\title{
BMJ Open Study protocol: Yarning about HPV Vaccination: a qualitative study of factors influencing HPV vaccination among Aboriginal and Torres Strait Islander adolescents in Australia
}

\author{
Lisa J Whop, ${ }^{1,2}$ Tamara L Butler (10 , 2 Julia M L Brotherton, ${ }^{3}$ Kate Anderson, ${ }^{2}$ \\ Joan Cunningham, ${ }^{2}$ Allison Tong, ${ }^{4}$ Gail Garvey (i) ${ }^{2}$
}

To cite: Whop LJ, Butler TL, Brotherton JML, et al. Study protocol: Yarning about HPV Vaccination: a qualitative study of factors influencing HPV vaccination among Aboriginal and Torres Strait Islander adolescents in Australia. BMJ Open 2021;11:e047890. doi:10.1136/ bmjopen-2020-047890

- Prepublication history for this paper is available online. To view these files, please visit the journal online (http://dx.doi org/10.1136/bmjopen-2020047890).

Received 11 December 2020 Accepted 21 June 2021

Check for updates

(C) Author(s) (or their employer(s)) 2021. Re-use permitted under CC BY-NC. No commercial re-use. See rights and permissions. Published by BMJ.

For numbered affiliations see end of article.

Correspondence to

Dr Lisa J Whop;

lisa.whop@anu.edu.au

\section{ABSTRACT}

Introduction Aboriginal and Torres Strait Islander women experience a higher burden of cervical cancer than non-Indigenous women in Australia. Cervical cancer is preventable partly through human papillomavirus (HPV) vaccination; in Australia, this is delivered through the national school-based immunisation programme. While HPV vaccination uptake is high among Australian adolescents, there remain gaps in uptake and completion among Aboriginal and Torres Strait Islander adolescents. This study aims to gain a comprehensive understanding of the barriers and facilitators to HPV vaccination uptake and completion among Aboriginal and Torres Strait Islander adolescents in Queensland, Australia.

Methods and analysis The study will be guided by an Indigenist research approach and an ecological model for health promotion. Yarning, a qualitative Indigenous research method, will be conducted in up to 10 schools. Participants will include Year 7 (12/13 years old) Aboriginal and Torres Strait Islander adolescents; parents/ caregivers; and local key informants and immunisation programme partners involved in the delivery of schoolbased HPV immunisation programme. Participants will be recruited through school representatives and investigator networks using purposive and snowball sampling and samples of convenience. Field notes, HPV vaccination clinic observations and sequential diagramming of the HPV vaccination process will be conducted. Thematic analysis of data will be led by Aboriginal and Torres Strait Islander researchers. Synthesised sequential diagrams of the process of HPV vaccination and qualitative themes summarising key findings will be produced.

Ethics and dissemination The Aboriginal Health and Medical Research Council of New South Wales Ethics Committee (1646/20), the Australian National University Human Research Ethics Committee (HREC, 2020/478), the HREC of the Northern Territory Department of Health and Menzies School of Health Research (19-3484) and the Townsville Hospital and Health Service HREC (HREC/ QTHS/73789) have approved the study. Dissemination will occur via conferences and peer-reviewed publications. Further dissemination will be determined in partnership with the Aboriginal and Torres Strait Islander Steering

\section{Strengths and limitations of this study}

- Using an Indigenist research approach, this study will prioritise the voices of Aboriginal and Torres Strait Islander people.

- Indigenous research methods will be used to triangulate the perspectives of Queensland Aboriginal and Torres Strait Islander adolescents, parents/ caregivers, local key informants and immunisation programme partners on human papillomavirus (HPV) vaccination.

- The methods will generate a comprehensive understanding of factors and processes affecting HPV vaccination uptake among Aboriginal and Torres Strait Islander adolescents

- A limitation is that the data are derived from only one Australian jurisdiction, the state of Queensland.

Committee, including Youth Representatives and Consultation Network.

\section{INTRODUCTION}

The WHO's vision to eliminate cervical cancer as a public health problem includes three main pillars: prevent, screen and treat. ${ }^{1}$ A key target to achieve this vision is to vaccinate at least $90 \%$ of females with human papillomavirus (HPV) vaccine by age $15 .^{1}$ Australia introduced primary prevention of HPV through a national school-based immunisation programme for females in 2007 and males in $2013 .^{2} \mathrm{HPV}$ vaccination is recommended by the National Immunisation Programme Schedule. ${ }^{3}$ The vaccine is free of charge through the school-based immunisation programme. The HPV vaccine is offered to all year seven students (first year of secondary school) along with a vaccine for diphtheria, tetanus and pertussis (dTpa). In general, paper-based consent forms are sent 
home with adolescents. Parents receive information and complete a consent form for each vaccine. Students who return a completed and signed consent form are vaccinated. Individuals 10-19 years of age are also eligible to receive the HPV vaccine free of charge via a catch-up programme outside the school-based immunisation programme, however, for adolescents $>15$ years, three doses are required, of which two are funded, and a consultation fee may be charged by the healthcare provider. ${ }^{4}$

Initially, the programme used a three-dose quadrivalent HPV vaccine (HPV types 6, 11, 16, 18) and in 2018 changed to a two-dose nonavalent HPV vaccine (HPV types $6,11,16,18,31,33,45,52$ and 58$).{ }^{2}$ The nonavalent prophylactic vaccine now covers seven cancer-causing HPV types including HPV genotypes 16 and 18, which are responsible for $70 \%$ of invasive cervical cancer cases. ${ }^{5}$ The vaccine also covers HPV types 6 and 11, which are responsible for $>90 \%$ of anogenital warts. ${ }^{6}$ HPV primary prevention through vaccination substantially reduces the risk of cervical cancer ${ }^{7}$ and has the potential to prevent up to $93 \%$ of cervical cancer cases in Australia. ${ }^{8}$ Australia previously set a target for $70 \%$ HPV vaccination coverage which was met in 2016 in both females and males. ${ }^{9}$ Subsequently, this target was increased to $80 \% .^{10}{ }^{11}$ Recent national data for the cohort of adolescents who turned 15 in 2019 demonstrate HPV vaccination coverage with completed courses in both sexes was $78.2 \%$ (79.6\% in females and $76.8 \%$ in males). ${ }^{12}$

Despite Australia having one of the world's lowest incidence rates of cervical cancer, ${ }^{13}$ Aboriginal and Torres Strait Islander women bear a greater burden of cervical cancer than other Australian women, demonstrated by the higher incidence, hospitalisation, and mortality rates, and lower survival rates. ${ }^{14}{ }^{15}$ In 2011-2015, Aboriginal and Torres Strait Islander women had twice the incidence of cervical cancer compared with non-Indigenous women (19.3 per 100000 vs 10.0 per 100000 women, respectively). Mortality rates in 2014-2018 are more than three times higher for Aboriginal and Torres Strait Islander women than non-Indigenous women (7.7 per 100000 vs 2.2 per 100000 women). ${ }^{16}$ Aboriginal and Torres Strait Islander women experience significant and persistent disparity in cervical screening participation and treatment outcomes. ${ }^{17-20}$ While national screening data are not available for Aboriginal and Torres Strait Islander women, localised studies have demonstrated that Aboriginal and Torres Strait Islander women's participation in cervical screening is significantly lower than non-Indigenous women. ${ }^{17}$ 21-23 In Queensland, the 2-year participation rate was more than 20 percentage points lower for Aboriginal and Torres Strait Islander women than for non-Indigenous women for all reporting periods examined from 2000-2001 to 2010-2011. In 2010-2011, 2-year participation was $33.5 \%$ for Aboriginal and Torres Strait Islander women and $55.7 \%$ for non-Indigenous women. ${ }^{17}$ This inequity in secondary prevention programmes for Aboriginal and Torres Strait Islander women highlights the urgency in ensuring equity in primary prevention via
HPV vaccination. It further speaks to the longstanding unacceptable racialised health disparities and disproportionate burden of disease, including cancer outcomes, experienced by Aboriginal and Torres Strait Islander peoples more broadly. ${ }^{24-26}$

National data on HPV vaccination uptake and coverage are not available for Aboriginal and Torres Strait Islander adolescents. Available data from four jurisdictions (New South Wales, Queensland, the Northern Territory and the Australian Capital Territory) for 2013-2016 indicate that among Aboriginal and Torres Strait Islander adolescents, Dose 1 vaccine coverage among females and males was high, ranging between $82.4 \%$ and $95.9 \%$. For the 2019 cohort, there was minimal variation in HPV vaccine uptake of Aboriginal and Torres Strait Islander adolescents by socioeconomic status or remoteness areas, with all groups exceeding $80 \%$ initiation rates, except for uptake rates among Aboriginal and Torres Strait Islander males in the lowest socioeconomic group $(79.8 \%) .{ }^{12}$ In 2016 course completion rates in the three-dose quadrivalent schedule were generally lower for Aboriginal and Torres Strait Islander adolescents (between $70.9 \%$ and $88.7 \%$ for females and $60 \%$ and $85.7 \%$ for males) compared with non-Indigenous adolescents (between $81.6 \%$ and $94.4 \%$ for females and $79 \%$ and $94.8 \%$ for males). ${ }^{27}$ These data demonstrate successes in Dose 1 uptake among Aboriginal and Torres Strait Islander adolescents. However, disparities in completion rates indicate that Aboriginal and Torres Strait Islander males and females are not receiving the same benefit from the full vaccination course as non-Indigenous adolescents. Importantly, these estimates represent the overall state/territory figures and may mask important geographical differences.

Little is known about: the factors that facilitated the relatively high uptake of Dose 1 and the barriers that impacted on course completion; the reasons for genderbased disparities between Aboriginal and Torres Strait Islander males and other groups and the availability, use of, and cultural appropriateness of, resources to support Aboriginal and Torres Strait Islander families' HPV vaccination decision-making. Furthermore, the school-based immunisation programme's capacity to be resilient to disruption is not documented or well understood-an important issue during the COVID-19 pandemic. These factors impact on the capacity of Queensland's schoolbased immunisation programme to be equitable, resilient and sustainable.

Barriers to HPV vaccination for Indigenous peoples in Canada, Aotearoa New Zealand and the USA span issues such as low awareness and understanding of HPV vaccination; limited information availability; access to clinics; cost of the vaccination; missing or incomplete data; ongoing impacts of colonisation including distrust of governments and health institutions; and concerns about the needle being painful. ${ }^{28}$ The COVID-19 pandemic has stalled HPV vaccination delivery. ${ }^{29}$ The factors that facilitate Indigenous peoples' access to HPV vaccination include family and community involvement in decision-making 
and health promotion materials and an explicit focus on equity in vaccination programme implementation. ${ }^{28}$ It is unclear if and how these factors feature in the Australian setting for HPV vaccination among Aboriginal and Torres Strait Islander people.

\section{Rationale and aims}

Effective and equitable primary prevention of HPV through a school-based immunisation programme has the potential to reduce the high incidence and mortality rates from cervical cancer and other HPV-related diseases. However, little is known about the specific barriers or facilitators to the delivery of HPV vaccination for Aboriginal and Torres Strait Islander adolescents via the existing school-based immunisation programme, nor the programme's ability to support access to HPV vaccination when school attendance is disrupted, making it difficult to identify and implement effective strategies to support and sustain equitable HPV vaccination. HPV vaccination was identified as a key priority in the National Aboriginal and Torres Strait Islander Cancer Framework ${ }^{30}$ which was underpinned by Aboriginal and Torres Strait Islander leadership and community consultation. ${ }^{30}$ In line with these identified priorities, Aboriginal and Torres Strait Islander researchers (Whop, Garvey, Butler) led the development of the study aims. Focused in the state of Queensland, the aims of this study are to:

1. Gain a comprehensive understanding of the barriers and enablers that influence the participation of Aboriginal and Torres Strait Islander adolescents in the school-based HPV vaccination programme.

2. Document the typical procedures in the school basedimmunisation programme through which vaccination of Aboriginal and Torres Strait Islander adolescents is achieved.

3. Understand the factors that impact on the resilience of the school-based immunisation model during the COVID-19 pandemic.

\section{METHODS AND ANALYSIS}

\section{Study design and approach}

Yarning about HPV Vaccination is a multi-component project; only the qualitative component is described here. A second component, not described here, will evaluate the informational and educational resources available to adolescents and parents/caregivers.

\section{Indigenist research approach}

The project will use Rigney's Indigenist research approach, ${ }^{31}{ }^{32}$ which is guided by three principles: (a) resistance as its emancipatory imperative, (b) political integrity in Aboriginal and Torres Strait Islander research, (c) privileging Aboriginal and Torres Strait Islander voices. Essentially, Indigenist research is: 'research by Indigenous people whose primary informants are Indigenous people and whose goals are to serve and inform the Indigenous struggle for self-determination' (p118). ${ }^{31}$ This approach ensures that the project aligns with the values of the Aboriginal and Torres Strait Islander communities it is intended to benefit.

Indigenist research principles ${ }^{31} 32$ will be operationalised in the study through strategies including: leadership, governance and yarns conducted by Aboriginal and Torres Strait Islander people; the use of yarning as a qualitative Indigenous research method; prioritising the views and lived experience of Aboriginal and Torres Strait Islander people; and the collaboration, support and partnership of non-Indigenous researchers who uphold Indigenist research principles.

\section{Ecological model for health promotion}

This approach is complemented by the ecological model for health promotion. ${ }^{33}$ This model views health-related behaviours, such as HPV vaccination, as an outcome of a complex interplay of intrapersonal, interpersonal, institutional, community and policy factors. This approach avoids victim-blaming when investigating health-related disparities and designing health promotion programmes. ${ }^{33}$ It has been successfully used as a framework to qualitatively identify and understand the barriers and facilitators to HPV vaccination in other populations. ${ }^{34}$

\section{Governance and consultation}

Patient and public involvement statement

As this protocol addresses a public health programme, no patients are to be recruited. We instead seek involvement of the relevant population for the school-based immunisation public health programme and public. Individuals from the relevant population and/or the public were not involved in the design, or conduct, or reporting, or dissemination plans for this study protocol. However, Aboriginal (Garvey, Butler) and Torres Strait Islander (Whop) researchers led the development of the study aims, study design and protocol, all of whom were living in the state of Queensland at the time of inception. Furthermore, HPV vaccination was identified as a key priority in the National Aboriginal and Torres Strait Islander Cancer Framework ${ }^{30}$ which was underpinned by Aboriginal and Torres Strait Islander leadership and community consultation. ${ }^{30}$ We intend to involve individuals from the relevant population and public in the design, conduct, reporting and dissemination of the research in the future, described later.

\section{Aboriginal and Torres Strait Islander Steering Committee}

We will establish an Aboriginal and Torres Strait Islander Project Steering Committee (henceforth, Steering Committee), in line with the Medical Journal of Australia guidelines ${ }^{35}$ and the principles of the Indigenist research approach. ${ }^{31} 32$ The Steering Committee will provide overarching project governance and ensure that the project is culturally safe and meets the needs and priorities of Aboriginal and Torres Strait Islander people and communities. Consultation with individual communities and schools will also be conducted to ensure the project is locally acceptable and feasible. 
All members of the Steering Committee will identify as Aboriginal and/or Torres Strait Islander people. The Steering Committee will include people with a range of expertise in areas such as Aboriginal and Torres Strait Islander Health, primary healthcare, vaccination, health workforce development, and parents/caregivers and school representatives from participating schools. A small number of Aboriginal and/or Torres Strait Islander people from other jurisdictions with relevant roles and experiences may also be invited to join the Steering Committee.

In line with the Cancer Australia Framework for Consumer Involvement in Cancer Control, ${ }^{36}$ at least two Aboriginal and/or Torres Strait Islander adolescent consumers will be invited to join the group. A Youth Representative Committee will be formed if four or more adolescents want to join the Steering Committee. The adolescent/s will be from previous Year 7 cohorts offered the HPV vaccine to avoid influence over decisions to have the vaccination.

\section{Consultation Network}

A Consultation Network will also be established, with the purpose of engaging key bodies involved in HPV vaccination to maximise uptake of the study findings and providing strategic advice in issues such as site recruitment. The research team will inform the Consultation Network of the study progress and preliminary findings. The Consultation Network will have Aboriginal and/or Torres Strait Islander representation. Representatives from peak Aboriginal and Torres Strait Islander health organisations and government health and education departments will be invited to join the Consultation Network. It may also include national representatives from other jurisdictions with expertise in school programmes and interests in supporting Aboriginal and Torres Strait Islander adolescents' HPV vaccination.

\section{Setting}

The state of Queensland is the second largest state in Australia and is situated in the north east of Australia. It is geographically vast and includes the islands of the Torres Strait. The second largest number of Aboriginal and Torres Strait Islander people live in Queensland. Approximately $4.6 \%$ of the total Queensland population identify as Aboriginal and/or Torres Strait Islander. ${ }^{37}$ Approximately $8.2 \%$ of 12-year-old and 13-year-old Queensland adolescents identify as Aboriginal and/or Torres Strait Islander. ${ }^{37}$

Up to 10 Queensland schools will be recruited into the study. After approval to approach the school principal about the research is granted by relevant governing body, the schools will be purposively sampled in line with the following eligibility criteria:

1. The school is in Queensland and has a Year 7 cohort.

2. The school has a relatively high proportion of Aboriginal and Torres Strait Islander students, defined as schools whose total Aboriginal and Torres Strait Island- er student enrolment is equal to or greater than $6 \%$. This proportion is slightly lower than the proportion of Queensland 12-year-old and 13-year-old Aboriginal and/or Torres Strait Islander adolescents to increase the pool of eligible schools that can potentially be recruited into the study.

3. The overall mix of school sites offers diversity in locations across Queensland, with respect to geographic remoteness (remote, regional and urban), school sector (government, Catholic or independent), student gender (single or mixed gender) and representation of residential boarding schools.

Special schools will not be eligible for the study as they provide highly specialised and individualised programmes that are not within the scope of the Yarning about HPV Vaccination project.

Eligible schools will initially be identified using the Australian Curriculum, Assessment and Reporting Authority School Profile data. ${ }^{38}$ School eligibility will be confirmed during the initial consultation with identified schools.

\section{Participants and recruitment}

Participants are outlined in table 1. In qualitative research, sampling aims to include information-rich cases and achieve depth of understanding rather than striving to meet a specific (statistically determined) sample size. ${ }^{39}$ Sampling will reach a diverse range of participants and achieve data saturation across themes. The sample size of each group has also been determined by practical considerations. These include: budgetary limits; practical timeframes of qualitative field work and reasonable workload requests for the school representative, particularly in the context of COVID-19 pandemic learning disruptions. ${ }^{40}$

School principals will be invited to discuss the study on behalf of the school. Should the school principal consent for their school to participate, they will identify a school representative to facilitate the study. The school representative will be the main liaison point between the research team and participants and will facilitate recruitment and scheduling of Aboriginal and Torres Strait Islander students and parents/caregiver yarns at each site. The school representative will be an individual working in a role that involves contact and communication with Aboriginal and Torres Strait Islander students and community, for example, an Aboriginal or Torres Strait Islander Teacher Aide, Education Support Officer or Community Liaison Officer already employed by the school. It could also be an Elder in residence at the school (a community member who is recognised as a holder of knowledge, cultural practice and lore). Alternatively, the school principal may nominate a staff member who will fulfil a similar role for the purpose of the study.

Principles of reciprocity and mutual benefit essential to research in Aboriginal and Torres Strait Islander communities ${ }^{41}$ will be enacted by negotiating services or activities that will benefit the school and the Aboriginal and Torres Strait Islander students (eg, presentations at 
Table 1 Summary of participants, sampling and recruitment

\begin{tabular}{|c|c|c|}
\hline Participant group & N (approximate) & Sampling and recruitment method \\
\hline $\begin{array}{l}\text { Year } 7 \text { Aboriginal and Torres Strait Islander } \\
\text { adolescents }\end{array}$ & 8-12 per school & $\begin{array}{l}\text { Convenience sampling via school representative. } \\
\text { Additional participants may be recruited via } \\
\text { community events outside the school. Purposive } \\
\text { sampling, as required, to include under- } \\
\text { represented groups (eg, adolescents who did not } \\
\text { receive vaccination) }\end{array}$ \\
\hline $\begin{array}{l}\text { Local key informants (eg, individuals from the school } \\
\text { involved in HPV vaccination programme delivery or } \\
\text { administering council) }\end{array}$ & 3-6 per school & $\begin{array}{l}\text { Purposive via principal and school representative, } \\
\text { and via local council administering HPV } \\
\text { vaccination delivery. Snowball sampling via } \\
\text { participants }\end{array}$ \\
\hline
\end{tabular}

HPV, human papillomavirus.

school events or providing light catering or activities at an event). Appropriate activities will be determined as part of the recruitment process.

\section{Adolescents and parents/caregivers}

School representatives will determine the most appropriate method of informing adolescents and parents/ caregivers of the study and seeking consent. We anticipate that most participants will be recruited via a study information and consent pack sent home to parents/ caregivers with students. The option for information and consent provided via email and/or online survey will also be offered. Parents/caregivers and adolescents may participate independently of each other (matched adolescent and parent/caregiver family groups are not required). Adolescents and parents/caregivers will be eligible to participate regardless of whether the parent/ caregiver consented for the adolescent to receive the HPV vaccination, or whether the adolescent received two, one or neither scheduled HPV vaccination.

Recruitment via schools may be supplemented with recruitment via community events and activities, such as sporting events and festivals, and via health services and land councils. This supplementary recruitment may reach adolescents who may not attend school frequently. Participants recruited in this setting (both adolescents and parents/caregivers) will be directly approached by the research team.

The school representative may also advertise the study where Aboriginal and Torres Strait Islander adolescents and their caregivers/parents may see it, for example, newsletters, online school forums or mailing/email lists. Finally, the research team will be flexible to the needs of each individual school regarding information dissemination about the project; for example, we may conduct a short information session prior to the school visit.
Local key informants and immunisation programme partners

Local key informants will be initially identified by the school principal and school representative. Participants may include individuals involved in HPV vaccination programme delivery both inside and outside the school, with additional participants identified via snowball recruitment. Individuals at local councils responsible for administering HPV vaccination at the school site will be invited to participate by the research team. Immunisation programme partners will be recruited purposively through investigator networks, the Consultation Network and snowball sampling. Additional research approvals to interview individuals outside the school setting will be obtained as necessary.

\section{Consent}

The school principal will provide written informed consent for their school to participate in the research, and optionally, for researchers to observe the HPV vaccination clinic in progress. All participants will provide informed consent prior to the yarn or yarning circles. When yarns are conducted over the phone or online, oral informed consent will be audio-recorded and transcribed. A parent/caregiver must provide consent for their child to participate in the study, and adolescents must also consent to participate.

\section{Data collection}

Several methods of qualitative data collection will be used in this study: yarning, construction of sequential diagrams and observation.

\section{Yarning}

Semistructured yarns and yarning circles will be used to explore participants' views and experiences in a culturally appropriate and safe manner. Male and female Aboriginal 
Table 2 Yarning topics

\begin{tabular}{ll} 
Participant group & Yarning topics \\
\hline Year 7 Aboriginal and Torres Strait Islander adolescents & HPV vaccination views and awareness. \\
Parents/caregivers of Year 7 Aboriginal and Torres Strait & Experience of HPV vaccination (adolescents only). \\
Islander adolescents & Impact of COVID-19 pandemic. \\
& Information and education about the vaccine. \\
& Improving HPV vaccination. \\
Local key informants (eg, individuals from the school & Role in HPV vaccination delivery. \\
involved in HPV vaccination programme delivery or & Creation and discussion of HPV vaccination process \\
administering council) & sequential diagrams. \\
& Information and education about the vaccine. \\
& Impact of COVID-19 pandemic. \\
Immunisation programme partners (eg, individuals from the & School's programme delivery. \\
state health and education departments involved in HPV & Role in HPV vaccination delivery. \\
vaccination programme) & Creation and discussion of HPV vaccination process \\
& sequential diagram.
\end{tabular}

HPV, human papillomavirus.

and/or Torres Strait Islander researchers trained and/ or experienced in yarning methodology will lead yarns with participants. Yarning involves conversational sharing of stories and information following cultural protocols. Yarning is generally more relaxed and informal than conventional interviews and establishes relationality between participant and researcher, which forms the basis of accountability and cultural safety between the two parties. A relationship between researcher and participant will be formed during the Social Yarning phase of the research. ${ }^{42}$ If deemed feasible and appropriate by the school representative, yarning circles may also be conducted, which involves facilitated yarning with a small group of people. Yarning circles are recommended as part of the Queensland Curriculum and Assessment Authority's resources for Aboriginal and Torres Strait Islander student perspectives. ${ }^{44}$ We will also seek the guidance of the school representative regarding whether yarning circles should be separated by gender.

Yarns may take place any time after Dose 1 or Dose 2 has occurred at the school site to avoid any conflation of the research with consent for vaccination. Yarns may take place in-person, online via virtual meeting software or over the phone. Yarns recruiting adolescents who may not attend school may also take place at community events and activities, such as sporting events and festivals or at health services and land councils. A semistructured yarning guide covering the topics listed in table 2 will be used, but questions will also be informed by observations and new topics raised in prior yarns. Where necessary and appropriate to the participant group, comparisons with other vaccination programmes, such as the concurrent dTpa vaccination, will be made to discern common and unique factors relating to
HPV vaccination uptake and completion. Adolescent and parents/caregiver participants will receive a gift card reimbursement for their time and contributions. At the conclusion of the yarn, researchers will provide adolescent and parent/caregiver participants brief information about HPV and the vaccination, and a brochure produced by the Australian Government and National Immunisation Programme with links to reliable information. ${ }^{45}$

All yarns will be audio recorded with the consent of the participant. Yarns will be transcribed verbatim by a transcription company. Participants who decline audio recording will be asked to consent to the researcher writing notes during the yarn.

The school representative will provide guidance on whether an Aboriginal and/or Torres Strait Islander interpreter will be required to support the yarns. Where appropriate, interpreters may conduct the yarns. The English interpretation of yarns conducted in language will be transcribed and analysed, with timestamps noted for non-English language.

During field work, researchers will take field notes to provide context for analysis. ${ }^{46}$ Field notes may include documenting contextual information about the school and research visit, sketches of locations or room layouts, emerging ideas to explore in forthcoming yarns and insight for data analysis. Other information will be noted as required. Field notes will be integrated with the data corpus to enrich analysis.

\section{Sequential diagramming}

During the yarns with local key informants and immunisation programme partners, participants and researchers will create sequential diagrams of their involvement in HPV 
vaccination at the school. ${ }^{47} 48$ Sequential diagrams (informally referred to as 'mud maps' with participants) elicit descriptions of processes or actions as chains of events. Initially, the participant will lead the construction of the diagram using either pen and paper or virtual meeting software drawing tools (participant-led diagramming), ${ }^{47}$ and then the researcher will explore significant events in the sequence and prompt for further information or key events in the process (researcher-led diagramming) ${ }^{47}$ Both the physical diagram and the verbal dialogue will be collected as data. The diagrams will both encourage qualitative discussion and contribute to the development of synthesised sequential diagrams of HPV vaccination for Aboriginal and Torres Strait Islander students at each school, as well as typical procedures state-wide.

\section{Observation of vaccination}

With the consent of the school principal, researchers will observe the HPV vaccination clinic (Dose 1 or Dose 2). A semistructured observation guide will be used, whereby researchers can provide both prompted and unstructured observations of how the clinic is conducted. Guided by previous research, ${ }^{49}$ observations may include sketches of the building and room layout, weather conditions, descriptions of significant events before, during and after vaccination, any emotional or physical reactions to the vaccination, descriptions of equipment, measures put in place as a result of COVID-19 (eg, social distancing) and any supports in place for Aboriginal and Torres Strait Islander students.

\section{Analysis}

Transcripts, field notes, sequential diagrams and observations will form the data corpus and will be managed with NVivo software (QSR International, V.12). ${ }^{50}$ Thematic analysis will be used to identify patterns of meaning across the data through an iterative process of coding, discussion and re-coding. ${ }^{51}$ A constructivist approach will be taken, in which experience and meanings are social reproductions and a product of sociocultural and structural conditions. ${ }^{51}$ In line with the Indigenist research approach, Aboriginal and Torres Strait Islander people will lead the initial coding and interpretation of the data, with support from non-Indigenous researchers. This approach brings a criticality to the interpretation and analysis of data afforded by Aboriginal and Torres Strait Islander people's lived experiences, relationality with participants and research expertise. Researchers will code the data line-by-line to identify emerging themes. The ecological model for health promotion ${ }^{33}$ will guide analysis, ensuring social, structural and environmental factors influencing HPV vaccination uptake are considered alongside individual level factors.

A sequential diagram of typical state-wide HPV vaccination practices will be synthesised by integrating the qualitative analysis findings, sequential diagrams and observational data and augmented by input from the investigator team, Consultation Network and Steering Committee.

\section{ETHICS}

Ethics approval for this research has been obtained from the Aboriginal Health and Medical Research Council of New South Wales Ethics Committee (1646/20), the Australian National University Human Research Ethics Committee (HREC, 2020/478), the HREC of the Northern Territory Department of Health and Menzies School of Health Research (19-3484) and the Townsville Hospital and Health Service HREC (HREC/QTHS/73789). We will adhere to COVID-19 health and safety guidelines in Queensland current at the time of fieldwork.

Participants will provide voluntary written or verbal informed consent. Adolescents will also require parent/ caregiver consent. School principals will provide consent for the school to participate and, optionally, for observational research. Participants may withdraw from the study at any time before dissemination of the findings, except for yarning circle participants, as it will be difficult to identify individual voices in the recording and transcript. Yarning circle participants will be informed of this before consenting to the study.

Data will be deidentified before publication. Some local key informants and immunisation programme partners may be identifiable due to the unique nature of their roles and this will be highlighted when seeking consent to participate. Yarning circle participants will be asked to respect other participants' privacy by not sharing information heard during the study.

Some participants may feel uncomfortable or embarrassed talking about the sexually transmitted nature of HPV or about needles; if this is the case, we will check the participant is happy to continue and/or change the topic. There is minimal risk of distress or harm due to participating in the research.

It will not be feasible to return to school sites to conduct member checking of interpretation with individual participants nor to seek participants' comment on yarning transcripts, however, findings will be discussed with the Steering Committee and Consultation Network to ensure they reflect the full range and depth of perspectives. The Steering Committee will include representatives of many communities involved in the research.

\section{DISSEMINATION}

Findings will be disseminated through conference presentations and peer-reviewed journal publications. Further dissemination will be determined in partnership with the Steering Committee and Consultation Network. Emphasis will be placed on meeting the Steering Committee Youth Representatives' recommendations. Dissemination will be flexible and responsive to each Committee's recommendations and needs. Expected dissemination formats include newsletters or summaries, short reports for 
schools and school principals, and social media. While this study will directly inform HPV vaccination knowledge and policy within the Queensland setting, we will disseminate findings to other relevant states/territory groups beyond Queensland to ensure applicable national-level strategy can be shared.

Publications will adhere to the CONSolIDated critERia for strengthening reporting of health research involving Indigenous Peoples (the CONSIDER statement) ${ }^{52}$ and the Consolidated Criteria for Reporting Qualitative Research. $^{53}$

\section{CONCLUSIONS}

The Yarning about HPV Vaccination project will comprehensively explore and document how multiple factors interact across interpersonal, institutional, community and policy levels to affect HPV vaccination for Aboriginal and Torres Strait Islander adolescents through an Indigenist research lens. This project will provide insights into increasing the resilience of the school-based immunisation programme during disruptions to school attendance, such as those caused by the COVID-19 pandemic. The findings may inform implementation strategies for other large-scale programmes such as the influenza vaccine and COVID-19 vaccine. The findings will be valuable to other countries seeking to implement equitable, resilient, accessible and sustainable school-based HPV vaccination.

Understanding the factors affecting HPV vaccination uptake among Aboriginal and Torres Strait Islander adolescents is a critical step in addressing the burden of cervical cancer among Aboriginal and Torres Strait Islander women. Equitable access to primary prevention through HPV vaccination has the potential to reduce disparities in cervical cancer outcomes for Aboriginal and Torres Strait Islander women in Australia.

\section{Author affiliations}

${ }^{1}$ National Centre for Epidemiology and Population Health, Australian National University, Canberra, Australian Capital Territory, Australia

${ }^{2}$ Menzies School of Health Research, Wellbeing and Preventable Chronic Diseases, Charles Darwin University, Casuarina, Northern Territory, Australia

${ }^{3}$ VCS Population Health, VCS Foundation, Melbourne, Victoria, Australia

${ }^{4}$ Sydney School of Public Health, The University of Sydney, Sydney, New South Wales, Australia

\section{Twitter Lisa J Whop @Lisa_J_Whop and Allison Tong @allisontong1}

Acknowledgements Ownership of Aboriginal and Torres Strait Islander knowledges and cultural heritage will be retained by the informant.

Contributors LJW, TLB, JMLB, KA, JC, AT and GG contributed to the conception of the study. LJW and TLB developed the detailed study methodology. TLB drafted the initial manuscript. LJW, TLB, JMLB, KA, JC, AT and GG contributed to developing the study design, provided feedback and reviewed drafts of the manuscript, and approved the final version.

Funding This project is supported by the Australian Research Council (ARC) (IN190100050) and the National Health and Medical Research Council (NHMRC) funded Centre of Research Excellence in Targeted Approaches To Improve Cancer Services for Aboriginal and Torres Strait Islander Australians (1153027). LJW was supported by an NHMRC Early Career Fellowship (1142035). TLB was supported by an ARC Discovery Australian Aboriginal and Torres Strait Islander Award (DAATSIA,
IN190100050) funded by the Australian Government. JMLB and KA received no specific funding for this work. JC was funded by an NHMRC Research Fellowship (1058244). AT was supported by an NHMRC Career Development Fellowship (1106716). GG was funded by an NHMRC Investigator Grant (1176651). The funders have no role in study design, data collection and analysis, decision to publish or preparation of the manuscript. The views expressed in this publication are those of the authors and do not necessarily reflect the views of the funders.

Competing interests None declared.

Patient and public involvement Patients and/or the public were not involved in the design, or conduct, or reporting, or dissemination plans of this research.

Patient consent for publication Not required.

Ethics approval Ethics approval for this research has been obtained from the Aboriginal Health and Medical Research Council of New South Wales Ethics Committee (1646/20), the Australian National University Human Research Ethics Committee (HREC, 2020/478), and the HREC of the Northern Territory Department of Health and Menzies School of Health Research (19-3484) and the Townsville Hospital and Health Service HREC (HREC/QTHS/73789).

Provenance and peer review Not commissioned; externally peer reviewed.

Open access This is an open access article distributed in accordance with the Creative Commons Attribution Non Commercial (CC BY-NC 4.0) license, which permits others to distribute, remix, adapt, build upon this work non-commercially, and license their derivative works on different terms, provided the original work is properly cited, appropriate credit is given, any changes made indicated, and the use is non-commercial. See: http://creativecommons.org/licenses/by-nc/4.0/.

\section{ORCID iDs}

Tamara L Butler http://orcid.org/0000-0001-6507-8157

Gail Garvey http://orcid.org/0000-0001-5065-5716

\section{REFERENCES}

1 WHO. Global strategy to accelerate the elimination of cervical cancer as a public health problem. Geneva: World Health Organization, 2020.

2 National Centre for Immunisation Research \& Surveillance. Significant events in human papillomavirus (HPV) vaccination practice in Australia 2018. Available: http://www.ncirs.org.au/sites/ default/files/2018-11/Human-papillomavirus-history-July-2018.pdf

3 Australian Government Department of Health. National immunisation program schedule 2021. Available: https://www.health.gov.au/ health-topics/immunisation/immunisation-throughout-life/nationalimmunisation-program-schedule

4 Australian Government Department of Health. Human papillomavirus (HPV) immunisation service 2019. Available: https://www.health. gov.au/health-topics/immunisation/immunisation-services/humanpapillomavirus-hpv-immunisation-service

5 de Sanjose S, Quint WG, Alemany L, et al. Human papillomavirus genotype Attribution in invasive cervical cancer: a retrospective cross-sectional worldwide study. Lancet Oncol 2010;11:1048-56.

6 Garland SM, Steben M, Sings HL, et al. Natural history of genital warts: analysis of the placebo arm of 2 randomized phase III trials of a quadrivalent human papillomavirus (types $6,11,16$, and 18) vaccine. J Infect Dis 2009;199:805-14.

7 Lei J, Ploner A, Elfström KM, et al. HPV vaccination and the risk of invasive cervical cancer. N Engl J Med 2020;383:1340-8.

8 Brotherton JML, Tabrizi SN, Phillips S, et al. Looking beyond human papillomavirus (HPV) genotype 16 and 18: defining HPV genotype distribution in cervical cancers in Australia prior to vaccination. Int $J$ Cancer 2017;141:1576-84.

9 Australian Government Department of Health. Third national sexually transmissible infections strategy 2014-2017, 2014. Department of health. Available: https://webarchive.nla.gov.au/awa/ 20180413214229/https://www.mbsonline.gov.au/internet/main/ publishing.nsf/Content/ohp-bbvs-sti.

10 Australian Government Department of Health. Fourth national sexually transmissible infections strategy 2018-2022. Canberra: Department of Health, 2018.

11 Australian Government Department of Health. Fifth national Aboriginal and Torres Strait Islander blood borne viruses and sexually transmissible infections strategy 2018-2022. Canberra: Department of Health, 2018.

12 NHMRC Centre of Research Excellence in Cervical Cancer Control. Australia's progress towards the elimination of cervical cancer as a public health problem: 2020 report. Melbourne, Australia, 2021. 
13 Arbyn M, Weiderpass E, Bruni L, et al. Estimates of incidence and mortality of cervical cancer in 2018: a worldwide analysis. Lancet Glob Health 2020;8:e191-203.

14 Australian Institute of Health and Welfare. Cancer in Aboriginal and Torres Strait Islander peoples of Australia: an overview. Canberra: AlHW, 2013.

15 Australian Institute of Health and Welfare. Cervical screening in Australia. 123 edn. Canberra: AlHW, 2019.

16 Australian Institute of Health and Welfare. National cervical screening program monitoring report. Canberra: AlHW, 2020.

17 Whop LJ, Garvey G, Baade P, et al. The first comprehensive report on Indigenous Australian women's inequalities in cervical screening: a retrospective registry cohort study in Queensland, Australia (20002011). Cancer 2016;122:1560-9.

18 Whop LJ, Baade P, Garvey G, et al. Cervical abnormalities are more common among Indigenous than other Australian women: a retrospective record-linkage study, 2000-2011. PLoS One 2016;11:e0150473.

19 Whop LJ, Baade PD, Brotherton JM, et al. Time to clinical investigation for Indigenous and non-Indigenous Queensland women after a high grade abnormal Pap smear, 2000-2009. Med J Aust 2017;206:73-7

20 Whop LJ, Bernardes CM, Kondalsamy-Chennakesavan S, et al. Indigenous Australians with non-small cell lung cancer or cervical cancer receive suboptimal treatment. Asia Pac J Clin Oncol 2017;13:e224-31.

21 Binns PL, Condon JR. Participation in cervical screening by Indigenous women in the Northern Territory: a longitudinal study. Med J Aust 2006;185:490-4.

22 Coory MD, Fagan PS, Muller JM, et al. Participation in cervical cancer screening by women in rural and remote Aboriginal and Torres Strait Islander communities in Queensland. Med J Aust 2002;177:544-7.

23 Dasgupta P, Aitken JF, Condon J, et al. Spatial and temporal variations in cervical cancer screening participation among Indigenous and non-Indigenous women, Queensland, Australia, 2008-2017. Cancer Epidemiol 2020;69:101849.

24 Australian Institute of Health and Welfare. The health and welfare of Australia's Aboriginal and Torres Strait Islander peoples 2015. Canberra: AlHW, 2015.

25 Vos T, Barker B, Begg S, et al. Burden of disease and injury in Aboriginal and Torres Strait Islander peoples: the Indigenous health gap. Int J Epidemiol 2009;38:470-7.

26 Australian Institute of Health and Welfare. Cancer in Aboriginal and Torres Strait Islander people of Australia. Canberra: AlHW, 2018.

27 Brotherton JM, Winch KL, Chappell G, et al. HPV vaccination coverage and course completion rates for Indigenous Australian adolescents, 2015. Med J Aust 2019;211:31-6.

28 Whop LJ, Smith MA, Butler TL, et al. Achieving cervical cancer elimination among Indigenous women. Preventative Medicine.2020;4.

29 Gilkey MB, Bednarczyk RA, Gerend MA, et al. Getting human papillomavirus vaccination back on track: protecting our national investment in human papillomavirus vaccination in the COVID-19 era. $J$ Adolesc Health 2020;67:633-4.

30 Australia C. National Aboriginal and Torres Strait Islander cancer framework. Surry Hills, NSW: Cancer Australia, 2015.

31 Rigney L-I. Internationalization of an Indigenous Anticolonial cultural critique of research methodologies: a guide to Indigenist research methodology and its principles. JSTOR 1999;14:109.

32 Rigney L. Indigenous Australian views on Knowledge production and Indigenist research. In: Kunnie J, Goduka I, eds. Indigenous Peoples Wisdom and Power: Affirming Our Knowledge Through Narratives. Burlington: Ashgate, 2006: 32-49.
33 McLeroy KR, Bibeau D, Steckler A, et al. An ecological perspective on health promotion programs. Health Educ Q 1988;15:351-77.

34 Ferrer HB, Trotter C, Hickman M, et al. Barriers and facilitators to HPV vaccination of young women in high-income countries: a qualitative systematic review and evidence synthesis. BMC Public Health 2014;14:700

35 Jamieson LM, Paradies YC, Eades S, et al. Ten principles relevant to health research among Indigenous Australian populations. Med J Aust 2012;197:16-18.

36 Cancer Australia and Cancer Voices Australia. National framework for consumer involvement in cancer control. In: Australia C, editor. Canberra, ACT, 2011.

37 Australian Bureau of Statistics. Estimates of Aboriginal and Torres Strait Islander Australians, June 2016. Canberra: ABS, 2018.

38 Australian Curriculum Assessment and Reporting Authority. ACARA data access program, 2020. Available: https://www.acara.edu.au/ contact-us/acara-data-access

39 Patton MQ. Qualitative research and evaluation methods. 3 edn. Thousand Oaks, CA: Sage Publications, 2002.

40 van Vonderen J. Queensland schools will be 'student-free' from next week amid coronavirus pandemic, Premier announces: ABC news, 2020. Available: https://www.abc.net.au/news/2020-03-26/ coronavirus-queensland-schools-to-be-student-free/12086568

41 National Health and Medical Research Council. Ethical conduct in research with Aboriginal and Torres Strait Islander peoples and communities: guidelines for researchers and stakeholders Canberra: Commonwealth of Australia, 2018

42 Bessarab D, Ng'andu B. Yarning about Yarning as a legitimate method in Indigenous research. International Journal of Critical Indigenous Studies 2010;3:37-50.

43 Walker M, Fredericks B, Mills K, et al. "Yarning" as a method for community-based health research with Indigenous women: the Indigenous Women's Wellness Research Program. Health Care Women Int 2014;35:1216-26.

44 Queensland Curriculum \& Assessment Authority. Yarning circles, 2018. Available: https://www.qcaa.qld.edu.au/about/k-12-policies/ aboriginal-torres-strait-islander-perspectives/resources/yarningcircles

45 Australian Government Department of Health. HPV vaccine brochure for Aboriginal and Torres Strait Islander peoples, 2018. Available: https://www.health.gov.au/resources/publications/hpvvaccine-brochure-for-aboriginal-and-torres-strait-islander-peoples

46 Phillippi J, Lauderdale J. A guide to field notes for qualitative research: context and conversation. Qual Health Res 2018;28:381-8.

47 Umoquit M, Tso P, Varga-Atkins T, et al. Diagrammatic elicitation: defining the use of diagrams in data collection. The Qualitative Report 2013;13:1-12.

48 Bravington A, King N. Putting graphic elicitation into practice: tools and typologies for the use of participant-led diagrams in qualitative research interviews. Qualitative Research 2019;19:506-23.

49 Bernard DM, Cooper Robbins SC, McCaffery KJ, et al. The domino effect: adolescent girls' response to human papillomavirus vaccination. Med J Aust 2011;194:297-300

50 International Q.S.R. NVivo qualitative data analysis software 2021.

51 Braun V, Clarke V. Using thematic analysis in psychology. Qual Res Psychol 2006;3:77-101.

52 Huria T, Palmer SC, Pitama S, et al. Consolidated criteria for strengthening reporting of health research involving Indigenous peoples: the consider statement. BMC Med Res Methodol 2019;19:173.

53 Tong A, Sainsbury P, Craig J. Consolidated criteria for reporting qualitative research (COREQ): a 32-item checklist for interviews and focus groups. Int J Qual Health Care 2007;19:349-57. 